\title{
REDUCTION OF UNDULATOR RADIATION AND FEL SMALL GAIN DUE TO WIGGLER ERRORS*
}

\author{
Aharon Friedman \\ National Synchrotron Light Source \\ Brookhaven National Laboratery \\ Upton, NY
}

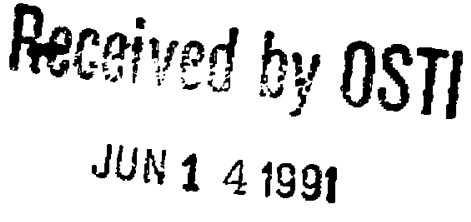

\begin{abstract}
A deterministic approech is taken to study the effect of errors in the wiggler magnet field on the spontaneous emission and the gain of Free Electron Lasers. A 3D formulation is used to derive the reduction in spontaneous emission due to changes in the time of flight of the electrons. A generalization of Madey's theorem to 3D is then used to calculate the reduction in the FEL small gain.
\end{abstract}

\section{INTRODUCTION}

The conventional approach taken in order to study the effect of wiggler errors on radiometric parameiers is statistical in its nature $[1,2]$. This approach is good at the Cesign stage of the device when tolerance must be specified for the construction of the wiggler. However, once the viggler is constructed, its errors can be measured and the radiometric parameters for that particular wiggler can be calculated. These parameters may differ significantly from those calculated at the design phase. A deterministic approach is, thus called for in order to evaluate the expected radiometric parameters and to decide how much effort is needed to correct the wiggler errors.

\section{REDUCTION IN SPONTANEOUS EMISSION (UNDULATOR RADIATION)}

The spectral radiant intensity of a charged particle is given by [3]:

$$
\frac{d P}{d \omega d \theta}=\frac{e \pi_{0}}{d x} \frac{1}{\lambda^{2}} \cos ^{2} \theta\left|I_{0}\right|^{2}
$$

where $\theta$ is the view angle and

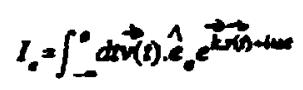

In equation $2 v(t), r(t)$ are the particle velocity and its respective trajectory $e_{,}, k$ are the polarization of the electromagnetic field and its wave vector respectively.

Work performed under the auspices of U.S. DOE.
In the wadulator approximation [3] the electron trajectory is assumed to be 2 straight line and the synchronization of the electron with the EM wave is through the trinsverse velocity imposed on it by the wigglex. For a highly relativistic electron this velocity is:

$$
\vec{\beta}=\frac{1}{\gamma} x[1+s(z)] \cos k, z \hat{e}_{x}
$$

where

$$
K=\frac{1}{2 \pi} \frac{e}{m c} B \lambda_{w}
$$

is the wiggler strength parnmeter and $s(z)$ is the error in the wiggler field. Substituting equation (4) into equetion (2) yields.

$$
I_{s}=\frac{\Gamma}{2 \gamma} e_{x} \cdot e_{0} \int_{0}^{2} \&[1+x(2)] e^{e s s}
$$

where

$$
\theta_{z}=\frac{2 \pi}{\omega}\left(\frac{1}{p_{z}}-\hat{\epsilon}_{k} \cdot \hat{\epsilon}_{i}-\frac{\lambda}{\lambda_{w}}\right)
$$

is the detuning parameter [4]. One can see that in equation (5) the effoct of the wiggler error on the rediation path integral I. can be distinguishod from the total path integral. Thus, the path integral may be writtea as:

$$
I_{a}=I_{\infty}+\Delta I_{c}
$$

where $I_{\infty}$ is the path integral of a perfect wiggler and $\Delta I$ is the contribution of the wiggler errors. Explicitly, the perfect wiggler path integral is:

$$
I_{\infty}=\frac{E}{2 \gamma} e_{x} \cdot e_{0} L^{\overline{n_{n}} \sin c} \frac{\bar{\theta}}{2}
$$

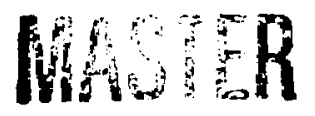


where

$$
\vec{\theta}=0, L
$$

and

$$
\operatorname{sinc} x=\frac{\sin x}{x}
$$

In order to calculate the wiggles error cortribution, it is assumed that $s(z)$ is constent along half a wiggler peziod of this assumption may not hold if the wiggler bes multi-poles per half perind). $S(z)$ can then be

$$
x(2)=s_{n} ; \text { for } n \frac{\lambda w}{2}<2(n+1) \frac{\lambda w}{2} ; 0<n<2 N
$$

In equation (11) $N=L / \lambda$, is the total number of wiggler periods. To simplify the calculation we assume that $s_{a}$ averages to zero over the wiggler length. Note that this assumption does not reduce the generality of the treatment, since the value of $\mathrm{K}$ can always be modified to accommodate it. Substituting equation (11) in samuation (5) results in:

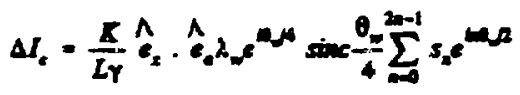

where

$$
\theta_{-}=\lambda_{-} \theta_{1}=\frac{\vec{\theta}}{N}
$$

We now can calculate the spectral radiant intensity by substituting equations $(8,9)$ into equation (1). Here again we express the total spectral radiant intensity as a sum of the radiation from a perfect wiggler and the modification of the wiggler errors.

$$
\frac{d P}{d \omega d Q}=\frac{d P_{0}}{d \omega d Q} \cdot \frac{d^{2}(\Delta P)}{d \omega d Q}
$$

It is preferable to exprese the wiggler errors term as a subtracting term, since one cen hardly expect thein to increase the radiation. The perfect undulator spectral radiant intensity is:

$$
\frac{d P_{0}}{d \omega d \theta}=\frac{e \eta_{0}}{16 \pi} \frac{I}{\lambda^{2}} \frac{\Sigma^{2}}{Y^{2}} \cos ^{2} \theta\left|\hat{e}_{x} \cdot \hat{e}_{e}\right|^{2} L^{2} \sin c \frac{\bar{\theta}}{2}
$$

The wiggler error term is

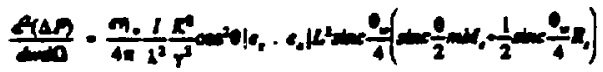

where

$$
H_{1}=\frac{1}{2 N} \sum_{n=0}^{2 n-1} s_{2} \sin ^{\theta} \frac{\theta}{4}\left(N-n-\frac{1}{2}\right)
$$

$$
R_{s}=\frac{1}{(2 N)^{2}} \sum_{n=0}^{2 n-1} \sum_{n=0}^{2 n-1} s_{n} s_{-\infty} \sin ^{2}(n-m) \frac{\theta_{0}}{4}
$$

It is constructive to calculate the relative reduction of radiation due to wiggler errors. This is achieved by dividing equation (16) by equation (15):

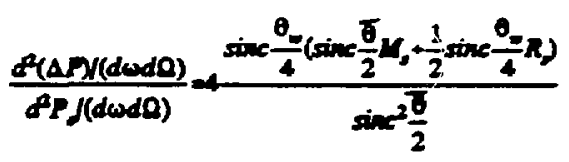

From equation (18) one can see that the relative reduction of spontaneous radiation of an undulator is independent on its length. The correlation functions $M$, and $R$, bave in them the total number of magnets poles. However, assuming that the errors have the same statistical behavior throughout the wiggler, this dependence cancels out. This fact leads to the important conclusion that one does not have to change the tolerances of a designed undulator when changing its length if its only purpese is to produce undulator radintion (e.g. an insertion device in a storage ring).

\section{REDUCTION IN SMALL GAIN}

The small gain in Free Electron Lasers can be calculated from the spontaneous emission of an undulator with the use of the generaiized Madey's Theorem. This Geoeralization of Madey's Theorem $[4,5]$ is described in reference [6]. In this paper the relation between spontaneous emission and gain was derived from first quantum electrodynamics principles and selection rules for electron-photon-wiggler interaction. The gain dependeace on the spontaneous spectral radinnt intensity is given in reference [6] by:

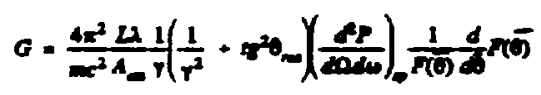

where the gain $G$ is defined as $G=\left(P_{E}-P_{i b}\right) / P_{i=}, A_{\text {man }}$ is the effective area of the electromagnetic mode being amplified and

$$
\cos \theta_{n c}=\hat{e}_{e} \cdot \frac{\vec{k}+\hat{e}_{z} k_{w}}{\left|\vec{k}+\hat{e}_{z} k_{w}\right|}
$$

is the recoil angle of the electron. In most practical cases $k_{w}<<k$, hence, $\cos \theta_{m \infty}-e_{.} \cdot e_{\mathbf{k}}$. In equation (19), $\mathbf{F}(\theta)$ is the lineshape function of the spontaneous emission. For a perfect 
wiggler this function is

$$
f(\bar{\theta})=\operatorname{sinc}^{2} \frac{\bar{\theta}}{2}
$$

Since the Generalized Madey's Theorem as described in equation (19) is based on Q.E.D. first principles, it is valid also for the case of a non-perfect wiggler. However, for a non-perfect wiggler the spontaneous emission can no longer be described as having a single lineshape function. Iristead, each terro in the sponteneous emission bas its own lineshape function. This problem can be ensily solved when one realizes that the derivative of the lineshape function in equation (20) is due to the fact that the emission and absorption control frequency are slightly displaced from each other. Thus, Madey's Theorem holds for each of the spontaneous emission terms independeatly. The total gain is, thus, the sum of the applications of Madey's Theorem to each of the spontaneous emission terms. The gain can also be written as the sum of the gain of a perfect wiggler minus wiggler errors contribution.

$$
G=G_{0}-\Delta G
$$

The gain of a perfect wiggler FEL is given in reference [6]

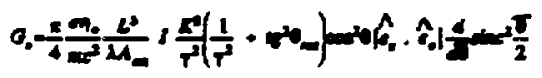

The reduction of the gain due to wiggler errors is calculated by applying equation (19) to eqs. (17).

$$
\begin{aligned}
\Delta G=\pi & \frac{e \eta_{0}}{m c^{2}} \frac{L^{2} \lambda_{w}}{\lambda A} I \frac{K^{2}}{\gamma^{3}}\left(\frac{1}{\gamma^{2}}+g^{2} \theta_{m c}\right) \cos ^{2} \theta\left|\hat{e}_{x} \cdot \hat{e}_{\sigma}\right|^{2} \\
& \times \operatorname{sinc} \frac{\theta_{\omega}}{4} \times\left(M_{G}+\frac{1}{2} \operatorname{sinc} \frac{\theta_{\sigma}}{4} R_{G}\right)
\end{aligned}
$$

In equations (25), it wes assumed that $N>>1$. It is agsin, interesting to calculate the relative roduction in gain:

$$
\frac{\Delta G}{G_{0}}=4 \frac{\lambda_{w}}{L} \frac{M_{G}+\frac{1}{2} R_{\sigma}}{\frac{d}{d \bar{\theta}} \sin ^{2} \frac{\bar{\theta}}{2}}
$$

Note that for a consistent siatistics of the wiggler poles the correlation functions $M_{G}$ and $R_{G}$ are expected to behave linearly in $N$. Thus, canceling the $\lambda \Omega$ factor in equation (26). Heace, the relatively reduction in gain is expected to be weakly coupled to the wiggler length.

\section{CONCLUSION}

The small gain and spontaneous emission reduction due to wiggler errors was calculated, including snas 3D effects. This formulation only takes into account the effect on the phase between the electron and the electromagnetic wave. However, there is another important effect to be taken into account that that is the effect of the "random walk" of the electrons from the axis. It seems, that the formulation used in this paper can be extended to include "random rnilk", and it is the intention of the author to pursue this course of action.

\section{REFERENCES}

1. B.M. Kincaid, Nuclear Inst. and Meth. Phys. Res. A-291 p.363 (1990).

2. J. Gallardo, BNL Report "45798 (1991).

3. A. Gover and A. Friedimn - to be published.

4. J.M.J. Madey, JAP 42, p. 1906 (1971).

5. S. Krinsky, J.M. Wang and P. Luchini, JAP 53, p.5453 (1982).

6. A. Friedmen, A. Gover, G. Kurizid, S. Ruschin and A. Yariv, Reviews of Modem Physics 60, 12, p. $\$ 71$ (1988).

where

$$
\begin{aligned}
M_{G} & =\frac{1}{2 N} \sum_{n=0}^{2 n-1} s_{n} \frac{d}{d \theta_{w}}\left[\sin \frac{M_{w}}{2} \sin ^{2} \frac{\theta_{w}}{4}\left(N-n-\frac{1}{2}\right)\right] \\
R & =\frac{1}{(2 N)^{2}} \sum_{n=0}^{2 n-1} \sum_{n=0}^{2 n-1} s_{N} s-\frac{d}{d \theta_{m}}\left[\sin ^{2}(n-m) \frac{\theta_{w}}{4}\right]
\end{aligned}
$$

\section{DISCLAIMER}

This report was prepared as an account of work sponsored by an agency of the United States Government. Neither the United States Government nor any agency thereof, nor any of their employees, makes any warranty, express or implied, or assumes any legal liability or responsibility for the accuracy, completeness, or usefulness of any information, apparatus, product, or process disclosed, or represents that its use would not infringe privately owned rights. Reference herein to any specific commercial product, process, or service by trade name, trademark, manufacturer, or otherwise does not necessarily constitute or imply its endorsement, recommendation, or favoring by the United States Government or any agency thereof. The views and opinions of authors expressed herein do not necessarily state or seflect those of the United States Government or any agency thereof. 